\title{
Deutsche Ophthalmologische Gesellschaft in Heidelberg
}

. Zusammenkunft in der Pfingstwoclie am 12., 13. und 14. Juni 1930.

I. Vorträge.

Amsler-L·ausAnne: Über Photo-Keratoskopie.

$\Lambda$ scher-Pra,g: Experimentelle Analyse der medikamentösen Augen-drucksenkung.

Baurmann- Göttingen: Klinische Beobachtungen bei zwei Fallen von Exophthalmus pulsans.

Bes $i$-Dresden: Die Kreuzung der sensorischen und motorischen Bah-nen und Cajals Theorie.

Blaickner-Salzburg: 1. Über Iridenkleisisoperationen. 2. Zur Be-handlung des Ulcus serpens. 3.

Zur Diagnose und Therapie der Linsenabszesse.

Deutsche Ophthalmologische Gesellschaft in Heidelberg. 273

Brückner-Iia.sel: Zur Regeneration der Glaskörperflüssigkeit.

Bücklers-E > e $\tau$ lin: Spektrographische Untersuchungen über die Absorption des Lichtes durch die menschliche Linse.

Comberg-'Berlin; Ein Faktor der Konvergenz und des Einwärts-schielens.

E/1/8es-Frankfurt a. M.: Experimentelle Studien über den Ablaut der Diphtherieinfektion am Auge und deren Beeinflussung durch Heil-serum.

10. jEwg $\cdot \mathrm{e} / \tilde{\mathrm{A}}^{1} / 8 \mathrm{~g}$ "-Freiburg i. Br.: Über individuelle Unterschiede im Farben-

sinn der anomalen Trichromaten. 12. Erggelel-Jena: Zur Frage der Strahlenwirkung am Auge.

Fischer-Leipzig: Ein Versuch, den Energiewechsel des Auges zu bestimmen.

Gilbert-Hamburg: Uvea und endokrine Störung.

Grẅ1/8r-Marburg: Die Atiologie der Keratitis disciformis.

Hartinger-Jena: Zur Berichtigung der Fehlsichtigkeiten mittels der geschliffenen Zeissschen

Haftgläser.

Herfe/-Leipzig: Weitere Studien über die Feinstruktur von Augen-geweben.

vom Hofe-L-eipzig: Untersuchungen über das Sehen in Fallen von Schielamblyopie.

Hoffmann-íïörngsberg i. Pr.: Netzhautveränderungen nach Röntgen-bestrahlung.

Jaensch-HzesİAu: Netzhautverfettung bei Endophthalmitis und sym-pathischer Ophthalmie.

Imre jun.-Budapest: Therapeutische $\Lambda 1 / 8$ rsuche und Erfolge mit Amyl-nitrit bei

Hintergrundserkrankungen.

Kubik-Ptag: Zur Pathologie menschlicher Starformen.

Kyrieleis-'Wiitzburg: Über die Wirkung des Strychnins auf einige Reflexe des Auges.

Lenz-'Bteslau: Untersuchungen über die innere Organisation der Sehrinde.

Lindner-Wien: Über meine derzeitige Technik der Goninschen Operation.

Loeffler-üied: Atmosphärische Unstetigkeitsschichten als auslösende Ursachen von akuten

Glaukomanfällen.

Löhlein-Jena: Die Bestimmung der Druckkurve am glaukomatösen Auge nach den Ergebnissen

ihrer 5jährigen systematischen An-wendung an der Jenaer Augenklinik.

Marchesani-München: Anatomische Untersuchungen von Netzhaut-gliomen.

Meesmann--Be $\tau$ lin: Über Blutgasanalysen am Kaninchenauge.

Meisner-Greifswald: Chronische Myositis der äußeren Augenmuskeln. 
Müller-Basel: Zur Messung des intraokularen Druckes.

My/îMS-Hamburg: Über Kammerwasser-Agglutinine.

Passow-München: Über die Bedeutung der Lipoide für das pri-märe Glaukom.

Pflimlin-Iia,sel: Zur klinischen Unterscheidung verschiedener For-men des Herpes corneae.

Poos-Münster i. W.: Morphologische Veränderungen bei pharmako-logischen Wirkungen am Auge. (Mit Demonstrationen.)

Peî ¿sc $\mathrm{Sc}^{3} / 8$-Hirschberg i. Rgb.: Über Kürzung der Bindehaut im Lid-spaltenteil.

î̂- $\mathrm{e} \mathrm{e}^{3} / 8 \mathrm{~W}$-Würzburg: Experimentelles zur Anaphylaxie - Forschung: a) Anaphylaxie und

Konstitution. b) Anaphylaxie und Trauma.

Rohrschneider--B > e tiin: Experimentelle Katarakt nach mehrfacher Bestrahlung mit kleinen

Röntgenstrahlen.

Scheerer-Tübingen: Über verschiedene Formen des Sehnervenaus-tritts, besonders bei

hochgradiger Kurzsichtigkeit.

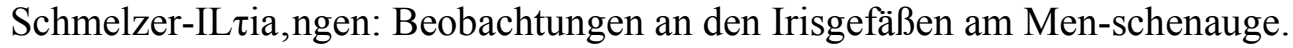

274 Deutsche Ophthalmologische Gesellschaft in Heidelberg.

Sondermann--Berlin: Beitrag zur Frage der Resorption aus der Vorder-kammer.

v. Szily-Münster i. W.: Herdreaktionen am Auge bei Einwirkung auf Kapillarendothelien. (Mit

Demonstrationen.)

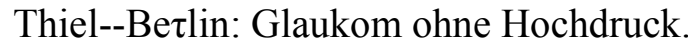

TA ¿es-Dessau: Innersekretorische Augenerkrankungen.

T homer--Rerliti: Ein Apparat zur stereoskopischen Ophthalmoskopie im rotfreien, fokalen und diffusen Licht.

Tschermak-Seysenegg-P $\tau$ ag: Was ist neutrales Licht?

Velhagen jun.-Halle a. S.: Untersuchungen über das Kammerwasser.

Vogelsang-üonn: Ballistische Elastometrie und Auge.

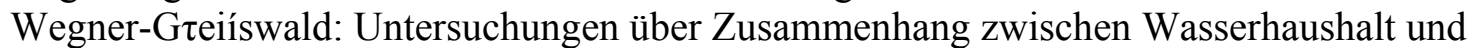

Glaukom.

Weräenberg-Oavos: Typischer und atypischer Krankheitsverlauf bei Augentuberkulose. (Mit

Demonstrationen.)

II. Demonstrationen.

Baurmann-Göttingen: Über das Ziliarfortsatzgefäßsystem.

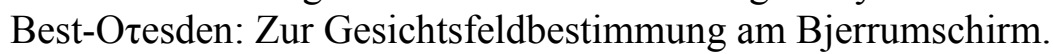

Brückner-E > asel: Zur Genese der Stauungspapille.

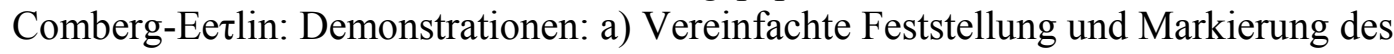

Operationsortes beim Netzhautriß. b) Quantitative Abschätzung einäugiger Schwachsichtigkeit mit Hilfe des Prismas.

Francescheíti und Müller--Basel: Zur Technik der Ophthalmoskopie im rotfreien Lichte.

Grüter-Maiburg: Zur Anatomie des Herpes corneae.

Guist-Wien: Demonstration eines Lokalisationsophthalmoskopes.

Hartinger-Jena. Ein neuès Photo-Keratoskop.

Hegner-Luze $\tau$ : Lesetafel für Naheproben.

Imre jun.-Budapest: Eine einfache Methode zur Ortsbestimmung des Netzhautloches bei der Goninschen Operation.

Junius--Boim•. Bild eines Falles von Angiomatosis retinae. 
Krückmann--Berlin: Einige Mitteilungen über den Frühjahrs-Katarrh.

Kyrieleis--WüTzbutg: Über multiple Ringtrübungen der Hornhaut nach Sprengverletzung.

Lindner-Wien: Demonstration einer erfolgreichen Impfung.

Löhlein-Jena: Bildersehprobe für die Nähe für Kinder und An-alphabeten.

Marchesani-München: Demonstrationen: a) Angeborenes Hornhaut-staphylom. b) v. Hippelsche Netzhauterkrankung.

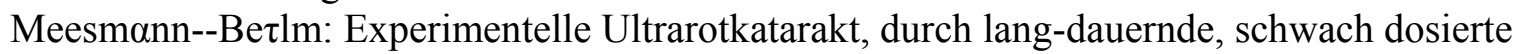
Bestrahlung erzielt.

Pascheff-Soíia: Demonstrationen: 1. Blepharospasmus sympathicus facialis. 2. Die spat entwickelten kornealen Wucherungen des Früh-jahrs-Katarrhs mit gleichzeitigem Symblepharon. i? ${ }^{1} 1 /{ }^{1} / 8$-Budapest: Infantile Heredodegeneration der Makulagegend in Form von Retinitis pigmentosa inversa.

Schreiber-iíeideibe tg: Zur pathologischen Anatomie der sympa-thischen Ophthalmie.

(Demonstration.)

$\mathrm{S}^{\wedge} \mathrm{M} \ll 1 / 8-H a m m:$ Demonstrationen: 1 . Ein neues Instrument zur sub-jektiven

Refraktionsbestimmung. 2. Eine neue Testmarke für Schei-telbrechwertmesser.

v. $S_{i i} / y$-Münster i. W.: Über Wandertuberkel im Auge.

TA ¿e/-Berlin: Photographierte Spaltlampenbilder.

Thies-Oessau: Der Verlauf einer schweren Verätzung in den ersten acht Tagen.

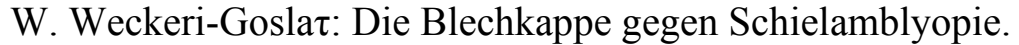

Za६\%o-Heidelberg: Demonstrationen: 1. Verband nach Staroperation. 2. Vorstichnadel zur Schieloperation. 3. Simulationsprüfung, 